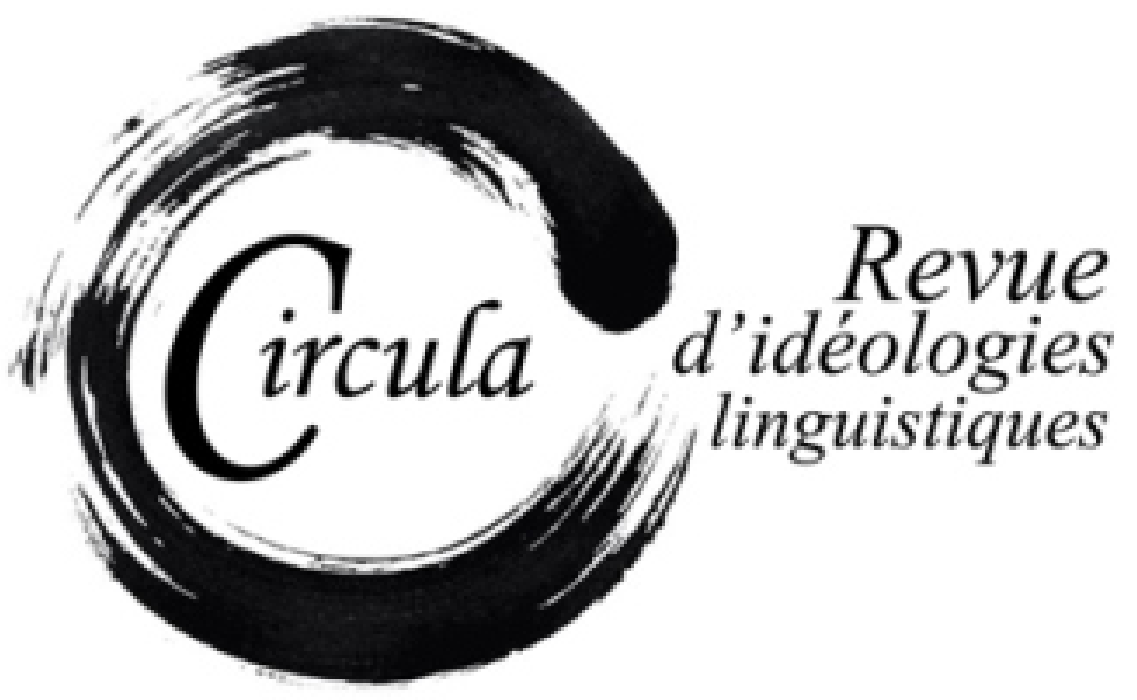

TITRE: ÉTUDE DIACHRONIQUE DU DISCOURS NORMATIF SUR LES ANGLICISMES DANS LES CHRONIQUES DE LANGUE AU CANADA FRANCOPHONE: D’ALPHONSE LUSIGNAN À GUY BERTRAND

Auteures: Mireille ElchacAr, Université TÉLUQ et AdA Luna SALITA, Université de SHerbrooke

Revue: Circula, numéro 9 : A-T-on encore PeUR des anglicismes? Perception actuelle des anglicismes au QuébeC ET DANS L'ESPACE FRANCOPHONE

PAGES: $4-28$

ISSN: 2369-6761

Directrices: MiREILle ElChaCAR ET NADINE VINCENT

URI: HTTP://HDL.HANDLE.NET/11143/16045

DOI: HTTPS://DOI.ORG/10.17118/11143/16045 


\title{
Étude diachronique du discours normatif sur les anglicismes dans les chroniques de langue au Canada francophone : d'Alphonse Lusignan à Guy Bertrand
}

\author{
Mireille Elchacar, Université TÉLUQ \\ melchaca@teluq.uquebec.ca
}

Ada Luna Salita, Université de Sherbrooke ada.luna.salita@usherbrooke.ca

Résumé : Cette étude se penche sur le traitement des anglicismes dans six chroniques de langue à visée normative publiées entre 1884 et 2018. Cent emplois commentés ont été analysés pour chaque chronique, pour un total de 600. Nous avons vérifié si une évolution dans le temps était perceptible pour les éléments suivants: dans quelles proportions les anglicismes font-ils l'objet d'un commentaire par rapport aux autres sujets abordés? Certains types d'anglicismes soulèvent-ils plus de questions que d'autres? Le jugement normatif sur les anglicismes est-il négatif, positif ou neutre? Quels arguments sont évoqués pour condamner un anglicisme ? II ressort de cette étude que les anglicismes demeurent un sujet de préoccupation normative chez les chroniqueurs de langue, les deux plus contemporains les abordant davantage que leurs prédécesseurs, et celui qui signe la chronique la plus récente les condamnant davantage que les autres du corpus.

Mots-clés : anglicismes; chroniques de langue ; étude diachronique

Abstract: This paper addresses the treatment of anglicisms in six normative language chronicles published between 1884 and 2018. One hundred comments were analyzed for each chronicle, for a total of 600 . We examined the following aspects: how many anglicisms are commented compared to all addressed topics? Are certain types of anglicisms more frequently mentioned than others? Is the normative judgement on anglicisms negative, positive or neutral? Which arguments are used to criticize an anglicism? We reached the conclusion that anglicisms remain a normative preoccupation for contemporary chronicles, the two most recent ones addressing them more than their predecessors.

Keywords: anglicisms ; language columns ; diachronic study 


\section{Introduction}

\subsection{La place des chroniques de langue au Canada francophone}

Vers la moitié du XIX ${ }^{\circledR}$ siècle, une campagne de rectification langagière lancée par les lettrés débute au Canada français; ; elle s'intensifiera au cours des décennies suivantes.

Dès la première moitié du XIXe siècle, le Canada français avait commencé à prendre conscience du fait que, pendant la période d'isolement qui avait suivi la Conquête, un certain écart s'était creusé entre le français du Canada et celui de France, notamment sous l'influence de l'anglais. Il avait également pris conscience de l'importance de son image linguistique dans le combat de résistance qu'il devait livrer aux pressions assimilatrices du Canada anglais. Ce double constat allait bientôt donner naissance à un mouvement de rectification langagière et provoquer l'apparition des premiers dictionnaires canadiens. (Mercier, 2008 : 61)

Cette campagne se manifeste par la publication de nombreux petits dictionnaires et autres ouvrages correctifs', et par la parution de chroniques de langue dans les journaux. Ces outils constituent des véhicules privilégiés du discours normatif sur la langue et de l'idéologie du standard, duquel le français au Canada s'éloignerait en raison et de sa forte anglicisation et de ses particularismes par rapport au français de Paris, décrit dans les dictionnaires courants et pris comme référence. On vise une correction de la langue française par un alignement sur le français de France : «Le but de l'entreprise normative était double : contrer l'influence de l'anglais par la chasse à l'anglicisme et enrichir le vocabulaire des Québécois, toujours en prenant appui sur la langue décrite dans les dictionnaires faits en France » (Mercier, Remysen, Cajolet-Laganière, 2017 : 298).

La lutte contre les anglicismes est omniprésente durant cette campagne : on fait un lien entre la mauvaise qualité de la langue chez les Canadiens francophones et la pénétration d'anglicismes dans leur parler. On pense par exemple à la conférence prononcée en 1880 par le journaliste Jules-Paul Tardivel, «L'anglicisme, voilà l'ennemi ! », qu'il conclut de la manière suivante:

Je m’arrête ici. Je ne prétends pas avoir épuisé le sujet, loin de là. J'ai voulu seulement jeter ce cri d'alarme: «L'anglicisme, voilà l'ennemi ! », et donner en même temps quelques preuves que cet ennemi est réellement à craindre. À d'autres plus autorisés que moi à continuer le combat, et si un jour ceux qui aiment la langue française se décident à livrer un grand assaut sur toute la ligne, soyez certains que je ne manquerai pas à l'appel. (Tardivel, $1880: 28$ )

Du côté des ouvrages correctifs, Manseau, auteur du Dictionnaire des locutions vicieuses du Canada avec leur correction, paru en 1881, juge les anglicismes dangereux:

1. Voir Mercier, 2008 pour une liste de ces titres. 
De toutes nos locutions vicieuses, [les anglicismes] sont certainement les plus dangereuses, parce qu'elles défigurent davantage la belle physionomie de notre langue; et plus humiliantes parce qu'elles impriment au front des cicatrices profondes qui semblent vouloir nous narguer et nous rappeler 1759 ! (Manseau, 1881 : III)

Tout au long de cette campagne, les chroniques de langue occupent une place importante et sont nombreuses. Le projet Chroqué : base de données textuelles constituée de chroniques québécoises de langage 2 , qui en a fait son objet d'études, a retenu comme étant importants et représentatifs de ce mouvement « 7936 billets correspondant à 68 chroniques différentes signées par une quarantaine d'auteurs de diverses époques et régions $»^{3}$.

Les jugements de tous ordres - et le plus souvent négatifs - auxquels la variété québécoise de français a été soumise depuis la deuxième moitié du XIXe siècle ont largement contribué à la construction de l'imaginaire linguistique des Québécois. Dans la diffusion de ces jugements, les chroniqueurs de langage ont joué un rôle de premier plan, aussi important que celui joué par les auteurs des innombrables ouvrages prescriptifs qui jalonnent l'histoire de la lexicographie québécoise, à qui les chroniqueurs ont du reste beaucoup emprunté tout en ne manquant pas de les alimenter en retour » (Gagné, Verreault et Mercier, 2004 : 247).

Le projet Chroqué a donné lieu à plusieurs recherches portant sur un aspect ou un autre de ces chroniques, dont la question des anglicismes. Les travaux de Prévost (1995) sur l'œuvre d'Etienne Blanchard mettent en lumière un discours normatif qui condamnait fortement l'anglicisme, non seulement comme menace à la langue, mais à l'identité canadienne française catholique ${ }^{4}$. Pellerin (2007), pour sa part, fait ressortir les bases argumentatives du discours de Louis-Philippe Geoffrion par rapport aux anglicismes : ce dernier dédie une partie de son travail à nier l'origine anglaise attribuée à certains emplois par ses collègues, mettant ainsi en garde contre les puristes qui voyaient de l'anglais partout. Remysen (2009) a travaillé sur l'imaginaire linguistique des chroniqueurs à partir de leur évaluation des canadianismes; sa thèse met en perspective les anglicismes par rapport aux autres préoccupations normatives des chroniqueurs. Sans s'inscrire dans le projet Chroqué, Bouchard (1989) s'intéresse à plusieurs aspects du discours médiatique sur l'anglicisme (dont le discours employé dans les chroniques de langue), entre autres, le vocabulaire péjoratif employé pour en parler, les attitudes envers l'anglicisme, ainsi que les solutions proposées pour le combattre. Enfin, Lamontagne (1996) s'est intéressée au discours sur l'anglicisme dans différentes sources métalinguistiques, principalement des dictionnaires de correction et certaines chroniques de langage, entre 1800 et 1930. Elle soulève qu'au début du XXe siècle, le terme anglicisme impliquait systématiquement une condamnation.

2. Pour plus d'information sur la base de données Chroqué, consulter le site Web qui y est consacré : https://catfran.flsh. usherbrooke.ca/chroque/

3. https://catfran.flsh.usherbrooke.ca/chroque/corpus_description.php

4. Rappelons que Blanchard était évêque. 
Même si les chroniques de langue sont moins répandues aujourd'hui, elles n'ont pas disparu du paysage médiatique. Guy Bertrand, premier conseiller linguistique de Radio-Canada, tient actuellement une chronique sur les ondes de la radio d'État francophone. Certains médias régionaux poursuivent la tradition, comme le journal sherbrookois La Tribune, où Steve Bergeron publie une chronique intitulée Séance d’orthographe.

Chantal Bouchard écrivait en 1989 : «il n’y a pas lieu de faire de distinctions chronologiques [entre les chroniques], car les auteurs portent sur les divers types d'anglicismes les mêmes jugements au XIXe siècle qu'en 1970 » (Bouchard, 1989 : 70). Nous avons voulu vérifier si cela est toujours le cas quelques décennies plus tard, surtout en ce qui concerne deux chroniqueurs contemporains, Paul Roux et Guy Bertrand.

\subsection{Objectifs}

Nous avons voulu étudier la place des anglicismes et les discours à leur sujet dans diverses chroniques de langue au Canada francophone, dans une perspective diachronique. Comme l'objectif du colloque auquel ces actes sont dédiés est d'étudier la perception actuelle des anglicismes, nous nous sommes penchées plus en profondeur sur deux chroniqueurs contemporains, soit Paul Roux et Guy Bertrand. Sachant que les discours sur la langue et sur les anglicismes ne sont pas homogènes et que des paroles discordantes peuvent coexister, au XIXe siècle comme aujourd'hui ${ }^{5}$, nous avons voulu voir comment des individus étant perçus comme porteurs de la norme envisagent et présentent les anglicismes, et comment les chroniqueurs plus contemporains se situent par rapport à ceux du passé.

\section{Les chroniques de langue analysées}

Définissons d'abord le sens avec lequel nous employons le mot «chronique »:

Le terme de chronique de langage désigne ici un ensemble de textes relativement brefs et homogènes, produits par une même personne (physique ou morale) reconnue pour sa compétence en matière de langue, diffusés périodiquement dans la presse et portant sur la langue, plus spécialement sur les bons et les mauvais usages qu’on en fait $[\ldots]^{6}$.

5. En réaction à un certain purisme véhiculé dans les chroniques à visée normative, et peut-être aussi à l'alignement sur le français de France, certains chroniqueurs adopteront une approche davantage descriptive. C'est le cas de Jean-Marie Laurence dans sa chronique « Notre français sur le vif » (1943-1947) ou de Philippe Barbaud dans sa chronique « Parler d'ici » (1984-1986) (voir Remysen 2009 pour plus de détails). On pense aussi à l'entreprise lexicographique de la Société du parler français, avec son Glossaire du parler français au Canada (voir Mercier, 2008).

6. Cette description est tirée du site web du projet Chroqué, consulté le 18 décembre 2018 : https://catfran.flsh.usherbrooke.ca/chroque/corpus_description.php. 
Nous avons analysé les chroniques produites par six personnes différentes de 1884 à aujourd'hui. Nous avons pris comme point de départ les deux plus récentes chroniques d'envergure nationale à avoir été publiées, celles de Paul Roux et Guy Bertrand. De 1991 à 2009, Paul Roux est conseiller linguistique et chroniqueur au quotidien La Presse. La chronique retenue est Mot du jour, publiée dans La Presse de 1996 à 1998. Guy Bertrand est présentement le premier conseiller linguistique à la radio d'État du Canada francophone, Radio-Canada. Sa chronique radiophonique, toujours diffusée au moment d'écrire ces lignes, s'appelle Le français au micro. Nous avons choisi d'analyser la chronique de Bertrand, même si elle est diffusée à la radio plutôt que publiée dans un journal, parce qu'il s'agit de la chronique actuelle qui jouit de la plus grande diffusion; en effet, elle est diffusée à travers le Canada francophone, et ce, de manière régulière (trois fois par semaine). Par ailleurs, elle présente le même format que les chroniques écrites, et la définition de chronique reproduite plus haut lui convient; seul le médium change. Afin d'observer l'évolution du discours sur les anglicismes dans le temps, nous avons sélectionné dans la base de données Chroqué des chroniques dont le format est comparable par la forme et le ton à celles de Roux et Bertrand. Ainsi, toutes les chroniques choisies adoptent une approche normative?

Nous avons tenté d'atteindre une certaine répartition dans le temps, de la fin du XIXe siècle à aujourd'hui, ce qui ne s'est pas avéré tout à fait possible en raison de nos critères de sélection, levolume des chroniques publiées augmentant considérablement uniquement à partir des années trente. Ainsi, pour la première moitié du XXe siècle, nous n'avons pas trouvé de chronique qui convienne exactement à nos critères de sélection. Nous avons tenté d'espacer les chroniques dans le temps et nous avons choisi des chroniques d'auteurs influents pour leur époque. La première chronique retenue est celle d'Alphonse Lusignan, secrétaire du ministère de la Justice et journaliste : «La langue française étant pour lui au cœur de l'identité de la nation canadienne-française, il était l'un de ses plus ardents défenseurs. » (Verreault, 2011) II publie la chronique Fautes à corriger. Une chaque jour dans La Patrie entre 1884 et 1885. Au tournant du siècle, nous avons retenu la chronique de Louis Fréchette intitulée À travers le dictionnaire et la grammaire, corrigeons-nous! publiée quotidiennement (La Presse, 18931903). " Destinée principalement à apporter des réponses aux questions de lecteurs qui faisaient appel à sa compétence en matière de langue française, cette chronique était également pour lui l'occasion de critiquer et de condamner la langue de ses confrères journalistes. » (Gagné et Verreault, 2011) Pour représenter le milieu du siècle, nous avons analysé la chronique Défense et illustration de la langue française (Le Droit, 1953-1963) de René de Chantal, qui était professeur de linguistique et littérature à l'Université de Montréal. Cette citation, tirée de sa chronique, illustre son point de vue sur l'approche nécessaire envers le français:

7. Nous avons donc écarté les chroniques de Daviault, qui sont davantage descriptives que prescriptives (voir Remysen, 2009), de même que celles de Barbaud ou Geoffrion mentionnées plus haut. Nous avons également mis de côté les chroniques de Blanchard, dont certains billets sont comparables à ceux de notre corpus, mais dont certains autres utilisent un format de type questions-réponses ou « dites-ne dites pas ». 
Notre parler franco-canadien ne peut continuer davantage son développement en vase clos; il doit faire face à l'anglais. Les résultats de cette confrontation ne se sont pas fait attendre: le vocabulaire d'abord, puis la syntaxe témoignent de l'influence de la langue anglaise. Bien des Canadiens estiment que l'avenir de notre langue est assuré parce que nous avons enfin obtenu, après quelles luttes, des garanties constitutionnelles. Erreur qui pourrait nous coûter cher, car c'est aujourd'hui que se livre la lutte dont l'enjeu est la survivance ou la disparition du français au Canada. (De Chantal, 16 mai 1956)

Finalement, nous avons retenu la chronique Comment dites-vous? (La Patrie, 1965-1966) de Gérard Dagenais, auteur de plusieurs chroniques et chroniqueur le plus influent de son époque. Dagenais adopte une attitude généralement assez puriste envers le français parlé au Canada :

Or je suis d'avis que nous ne parlons pas français. [...] « Notre français » souffre de plusieurs maladies graves : le barbarisme et le solécisme, auxquels aucune autre langue n'échappe, et l'anglicisme, dont on commence à souffrir en France ; elles ne sont graves au Canada que par leur virulence. (Dagenais, 1959)

Le schéma suivant illustre les chroniqueurs retenus et leur répartition dans le temps ${ }^{8}$ :

\begin{tabular}{|c|c|c|c|c|c|}
\hline & & De Chantal & & Roux & \\
\hline$\bullet 1884$ & & •1953 & & •1996-98 & \\
\hline \multirow[t]{3}{*}{0} & O & O & 0 & 0 & O \\
\hline & FrÉCHETTE & & DAGENAIS & & BERTRAND \\
\hline & •1899 & & •1965 & & •2017-2018 \\
\hline
\end{tabular}

\section{Schéma 1. Les chroniqueurs retenus}

Nous avons trouvé les chroniques de Paul Roux sur la base de données journalistique Eureka. Pour ce qui est de Guy Bertrand, des transcriptions de ses chroniques sont versées chaque semaine sur le site Internet du « Français au micro ${ }^{9}$ », puis celles qui ont plus de trois ou quatre mois sont effacées. Les autres chroniques sont sur la base de données Chroqué.

8. De plus amples informations sur les chroniqueurs (sauf De Chantal) sont disponibles sur le site de la base de données Chroqué : https://catfran.flsh.usherbrooke.ca/chroque/chroniqueurs_beaudry.php

9. http://linguistique.radio-canada.ca/category/le-francais-au-micro/ 
Chaque chronique est divisée en billets ${ }^{10}$, qui représentent la publication mensuelle, hebdomadaire ou quotidienne. Chacun des billets peut contenir un commentaire sur plusieurs emplois, que ce soit un anglicisme, un archaïsme, une tournure syntaxique, etc. Nous avons analysé 600 emplois commentés, c'est-à-dire 100 emplois commentés pour chacun des six chroniqueurs retenus. Pour Lusignan, De Chantal, Dagenais et Roux, nous avons commencé à récolter les billets à partir du début de la publication de leur chronique. Pour Fréchette, nous avons commencé le dépouillement à partir de 1899, afin de couvrir le tournant du siècle - sa chronique avait commencé à être publiée quelques années plus tôt. Enfin, les chroniques de Bertrand que nous avons choisies sont les plus récentes qui étaient disponibles au moment de mener la recherche.

Nous nous sommes intéressées à quatre aspects du discours des chroniqueurs : la proportion d'anglicismes parmi les emplois qu'ils commentent, le type d'anglicismes commentés, les prises de position normatives et les arguments employés pour justifier ces prises de position.

\section{Les analyses}

\subsection{Proportion d'anglicismes}

Pour les besoins de notre étude, nous avons analysé les commentaires qui portaient sur un usage explicitement reconnu comme influencé de l'anglais par le chroniqueur. Nous avons donc écarté les emplois condamnés où l'influence de l'anglais n'est pas clairement mentionnée ${ }^{11}$.

Nous avons d'abord chiffré la proportion d'anglicismes : sur les 100 emplois commentés retenus pour chaque chroniqueur, combien portent sur des anglicismes? Le schéma 2 illustre la place que prend l'anglicisme parmi les emplois commentés.

10. Nous reprenons la terminologie employée par l'équipe de Chroqué : « pour désigner chacun des textes constitutifs d'un même ensemble, nous utilisons le terme de billet. » https://catfran.flsh.usherbrooke.ca/chroque/corpus_description.php_

11. Par exemple, même si l'emploi « Seven up flat» ("Elle ne se prend pas pour un Seven Up flat! »), commenté par Bertrand, est vraisemblablement un emprunt à l'anglais, nous ne l'avons pas conservé dans notre corpus parce que l'auteur n'indique pas clairement qu'il le considère comme tel. Ce point de méthodologie est particulièrement utile pour les emplois qui sont moins facilement classables en tant qu'anglicismes par les non-spécialistes, comme «Elle a parti le projet en 2015 », autre cas tiré des chroniques de Bertrand. 


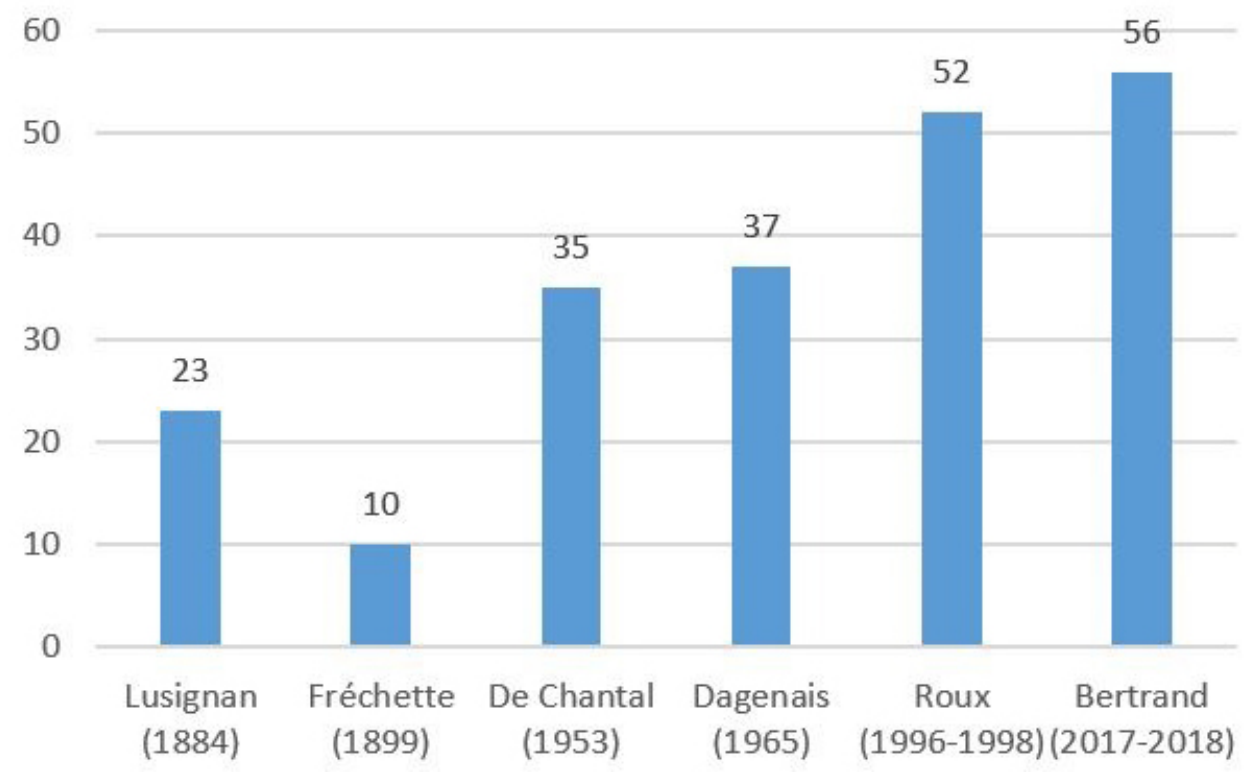

\section{Schéma 2. Nombre d'anglicismes parmi les emplois commentés}

En tout, sur les 600 emplois commentés analysés, 213 portent sur des anglicismes. Mis à part les données sur Fréchette ${ }^{12}$, on constate que plus on avance dans le temps, plus les anglicismes font partie des préoccupations des chroniqueurs, celui qui les aborde le plus étant Guy Bertrand, dont la chronique est la plus récente de notre corpus. Est-ce leur préoccupation ou celle du public ? Il est difficile de le savoir ; en effet, les chroniqueurs puisent leur inspiration à diverses sources. Certains répondent à des questions des lecteurs (mais peut-être opèrent-ils une sélection parmi les questions envoyées) ; Bertrand repère des écarts à la norme que ses collègues ont prononcé en ondes; d'autres enfin ne donnent pas d'information sur la manière dont leur choix s'est fixé ${ }^{13}$.

12. La proportion particulièrement basse de commentaires sur les anglicismes formulés par Fréchette pourrait s'expliquer par le fait qu'il commente généralement beaucoup d'emplois dans un même billet. Nous avons donc atteint le nombre de 100 emplois commentés en peu de billets, et il se peut que cela ne soit pas représentatif de toutes les préoccupations normatives qu'il aborde.

13. Remysen et De la Fontaine (2013) ont identifié le Glossaire du parler français comme source d'influence chez certains chroniqueurs. 


\subsection{Types d'anglicismes}

Nous avons ensuite examiné quels types d'anglicismes étaient le plus souvent commentés par les chroniqueurs.

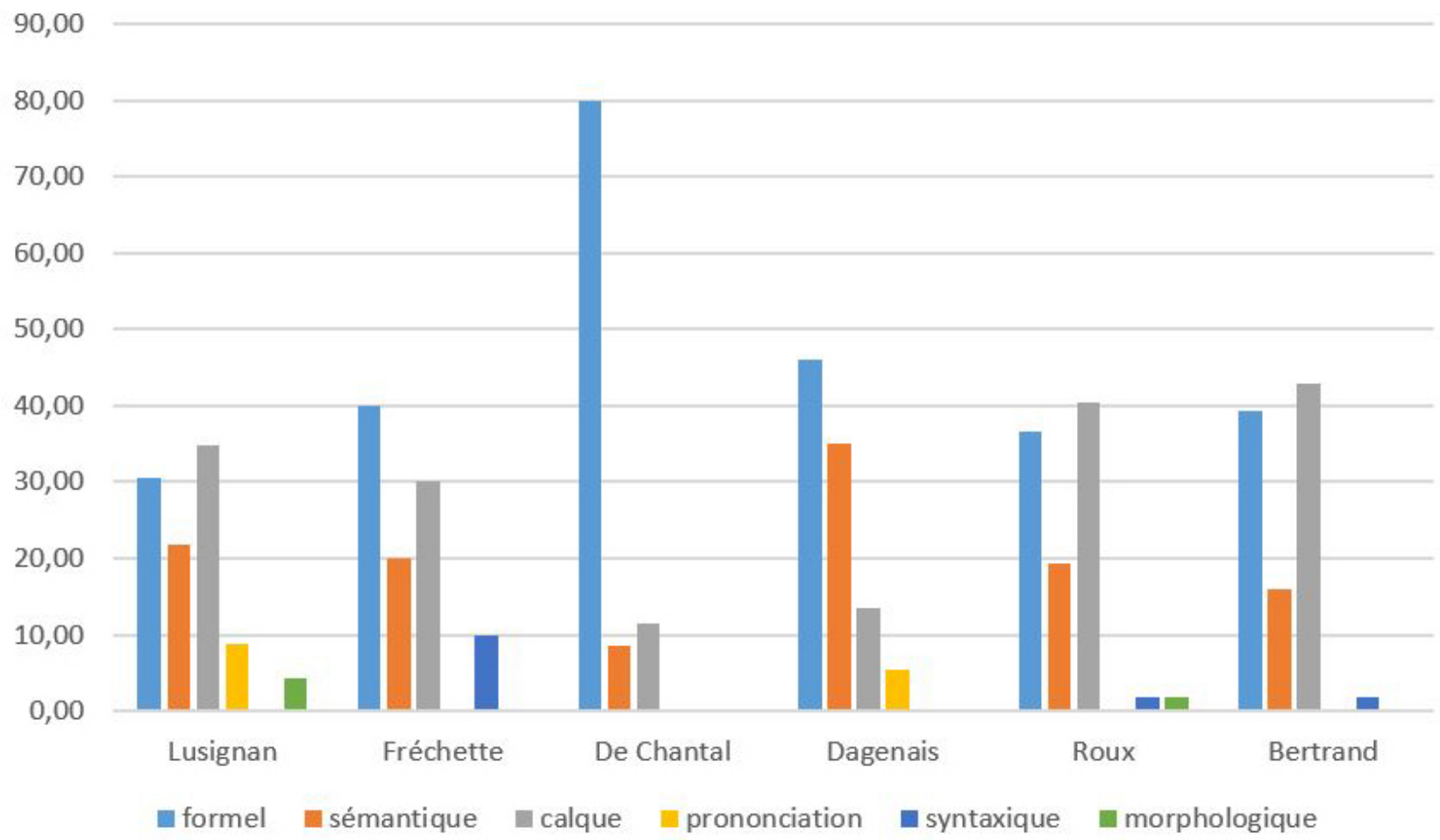

Schéma 3. Types d'anglicismes commentés

Les deux types d'anglicismes dont il est le plus souvent question sont les anglicismes formels (la forme en entier est empruntée à l'anglais ; le signifiant n'existait pas en français) et les anglicismes sémantiques (un sens est emprunté à l'anglais et s'ajoute au sens français qui était déjà associé au signifiant français), suivis par les calques (locutions ou expressions traduites littéralement de l'anglais). Le tableau 1 présente quelques exemples de ces types d'anglicismes tirés de notre corpus : 
Tableau 1. Exemples d'anglicismes formels, d'anglicismes sémantiques et de calques tirés des chroniques de langue

\begin{tabular}{|c|c|c|}
\hline Type d'anglicisme & Exemples & Chroniqueur \\
\hline \multirow{4}{*}{ Anglicisme formel } & pickles & Fréchette \\
\hline & véganisme & Bertrand \\
\hline & slip & De Chantal \\
\hline & sitcom & Roux \\
\hline \multirow{4}{*}{ Anglicisme sémantique } & banque (au sens de « tirelire ») & Roux \\
\hline & $\begin{array}{c}\text { agenda (au sens d' « ordre du } \\
\text { jour ») }\end{array}$ & Bertrand \\
\hline & $\begin{array}{c}\text { littérature (au sens de « docu- } \\
\text { mentation ») }\end{array}$ & Dagenais \\
\hline & apologie (au sens d'« excuses ») & Lusignan \\
\hline \multirow{3}{*}{ Calque } & manquer quelqu'un & Lusignan \\
\hline & prendre un cours & Dagenais \\
\hline & hors d'ordre & Roux \\
\hline
\end{tabular}

Nous trouvons aussi quelques cas d'anglicismes de prononciation (comme carbon et cantaloupe chez Dagenais), d'anglicismes morphologiques (comme l'utilisation d'argent au pluriel, critiquée par Roux) et d'anglicismes syntaxiques (Fréchette critique l'emploi de la préposition sur dans l'expression «sur la rue»). Ces types d'anglicismes sont toutefois beaucoup moins nombreux que les trois types présentés précédemment comme on peut l’observer dans le schéma 4 :

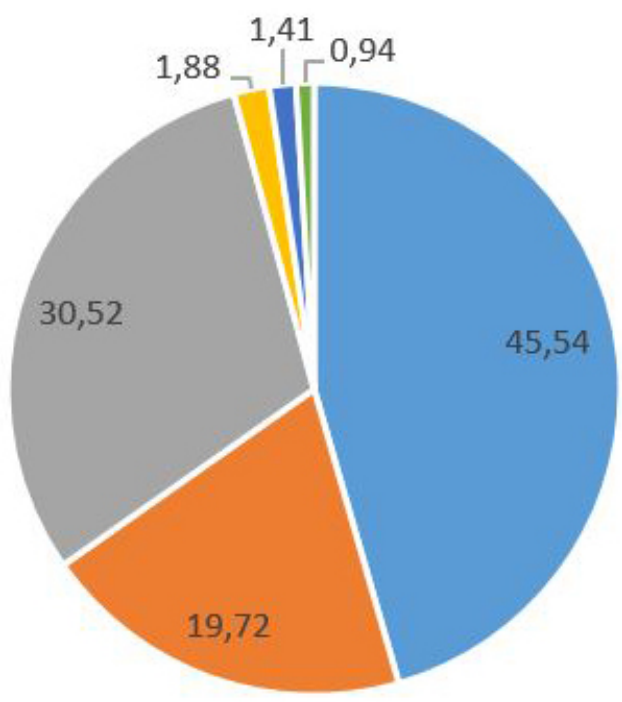

- formel = sémantique " calque $\mid$ prononciation " syntaxique " morphologique

Schéma 4. Types d’anglicismes commentés au total (en pourcentage) 
Donc, mis ensemble, les anglicismes morphologiques, syntaxiques et de prononciation ne représentent que 4,23\% des anglicismes commentés. Le type d'anglicisme le plus commenté est l'anglicisme formel (45,54 \% des cas), qui est le type d'anglicisme le plus visible et donc qu'on dénonce le plus au Canada francophone (Bouchard, 1999 : 22). On n'observe donc pas de changement dans le temps pour le type d'anglicisme commenté.

\subsection{Prise de position normative}

Nous avons ensuite analysé le jugement porté sur les anglicismes par les chroniqueurs. Ces derniers peuvent:

- accepter l'emploi d'un anglicisme :

(1) «Chemin normal traduit le mot anglais «fairway» [...] mais il n'est pas fautif de dire fairway (prononcer féroué). » (Dagenais, semaine du 14 nov. 1965)

- le condamner:

(2) « les locutions coûter un bras et coûter un bras et une jambe [...] sont des calques de l'anglais et leur emploi n'est pas recommandé. » (Bertrand, semaine du 16 oct. 2017)

- ou encore simplement donner de l'information, sans porter de jugement normatif:

(3) «Les mots skin et punk sont d'origine anglo-américaine. Ils désignent l'un et l'autre de jeunes marginaux» (Roux, 28 janv. 1998) 
Le schéma 5 montre la prise de position normative de chaque chroniqueur sur les anglicismes commentés:

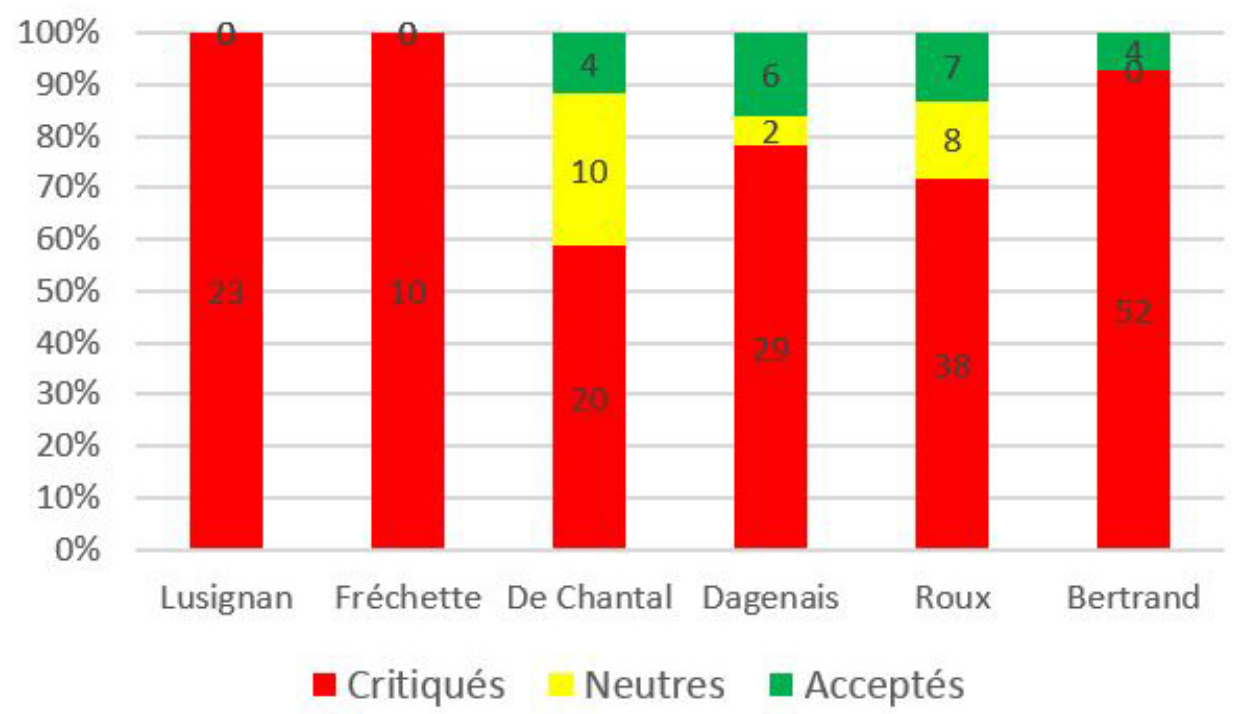

\section{Schéma 5. Prise de position normative sur les anglicismes}

Sur les 213 anglicismes commentés, 172 sont condamnés par les chroniqueurs, ce qui fait $80 \%$. Peu de commentaires sont neutres, et encore moins d'anglicismes sont jugés acceptables. Dans les deux chroniques les plus anciennes, celles de Lusignan et Fréchette, 100 \% des anglicismes sont critiqués : on est à la fin du XIXe siècle, avant les temps forts du mouvement glossairiste, qui amène une nouvelle manière de considérer les canadianismes, de façon davantage descriptive, et qui «se démarquera rapidement du discours puriste » (Mercier, 2008 : 63). Dans le cas de Dagenais, tous les anglicismes acceptés se trouvent dans un billet qui porte sur le vocabulaire du golf. Le taux d'acceptation peut donc être en lien avec ce vocabulaire spécifique. En effet, Dagenais « n'a qu'un seul objectif en tête : celui de montrer aux Québécois comment on parle et écrit en France » (Remysen et Tremblay-Desrochers, 2011). Or justement, plusieurs mots du vocabulaire sportif ont été empruntés par la France à l'anglais britannique durant la période d'anglomanie du XIXe siècle, et ne sont pas critiqués $^{14}$ :

Après 1815, on constate une nouvelle vague d'anglomanie, laquelle culminera au milieu du XIX siècle avec l'irruption massive de la terminologie anglaise dans les sports. Dans ce domaine, le français emprunte massivement à l'anglais pour la simple raison que c'est en Grande-Bretagne que se pratiquent bon nombre d'activités sportives et, surtout, parce que c'est dans ce pays que les règles et les pratiques sont fixées. (Loubier, 2011 : 23)

14. On peut voir un parallèle avec les emprunts faits à l'anglais dans le vocabulaire politique (Steuckardt, 2008). 
Quant à De Chantal, il utilise un ton neutre lorsqu'il évoque les anglicismes du vocabulaire du bridge, un jeu d'origine russe mais popularisé par des peuples de langue anglaise ${ }^{15}$, qu'il aborde dans un seul billet.

Si on examine la teneur de la critique envers les anglicismes, on constate que la plupart des jugements négatifs sont catégoriques plutôt que nuancés. Nous avons classé un jugement comme étant catégorique lorsque le chroniqueur le condamne sans appel:

(4) «Bien qu'ils soient répandus dans toute la francophonie, les calques de l'anglais tester positif et être testé positif sont toujours considérés comme abusifs. » (Bertrand, semaine du 4 décembre 2017)

Au contraire, nous avons classé un jugement comme étant nuancé lorsque le chroniqueur ne tranche pas clairement, ou fait état de plusieurs prises de position :

(5) « Le mot «pickles» peut se traduire par conserves au vinaigre. Mais, pour abréger, les Français ont adopté «pickles». On le trouve dans Edmond About, qui était bien puriste, pourtant. » (Fréchette, 28 oct. 1899)

Le schéma 6 illustre le nombre de jugements catégoriques et nuancés de notre corpus:

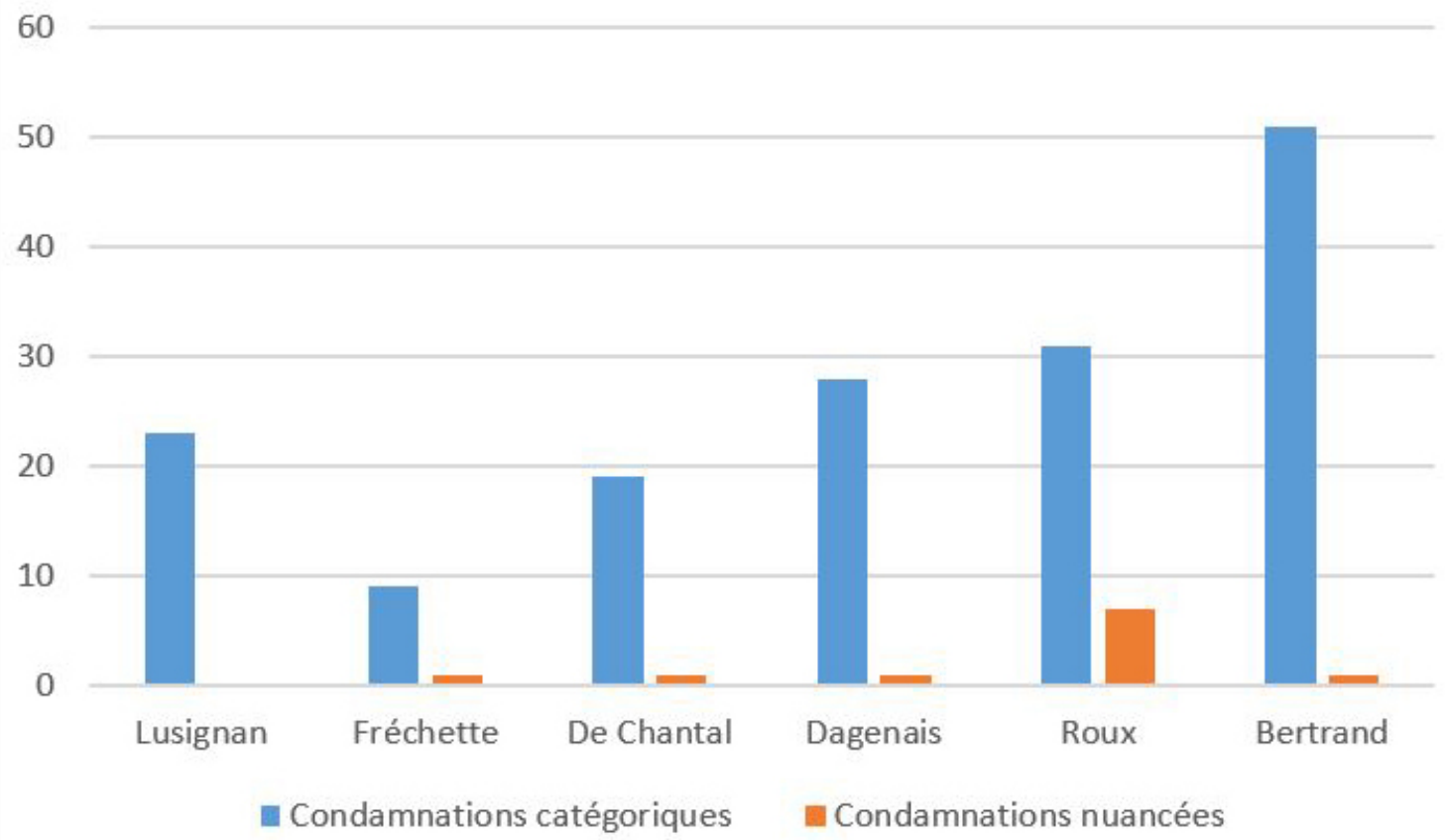

Schéma 6. Jugements catégoriques ou nuancés envers les anglicismes critiqués

15. Voir l'étymologie donnée dans le Trésor de la langue française informatisé : « Ce jeu d’orig. russe gagna Constantinople (1873), Athènes, Alexandrie, l'Angleterre (1875), puis Nice, d'où il passa aux États-Unis pour revenir en France (FEW; BONN.) » http://stella.atilf.fr/Dendien/scripts/tlfiv5/visusel.exe?11;s=2155871745;r=1;nat=;sol=0;. 
Le chroniqueur qui apporte le plus de nuance dans ses critiques est Roux, comme dans ce billet sur sitcom:

(6) «Ce terme est l'abréviation anglaise de situation comedy. On peut donc le traduire par comédie de situation, comme le fait d'ailleurs le Harrap's. [...] Cependant, on rencontre sitcom de plus en plus souvent, tant chez nous qu'en France. Mais son sens ne paraît pas toujours clair, comme en témoigne cet emploi dans une revue française, où l'on parle d'un sitcom comique. Il est vrai que certaines de ces comédies ne sont pas très drôles. » (Roux, 19 juillet 1998)

Roux est tout de même majoritairement catégorique à l'heure de critiquer un emploi. Les autres chroniqueurs sont presque toujours catégoriques dans leur condamnation d'un anglicisme:

(7) «Le verbe HARNACHER n'a pas d'autre sens que celui de «mettre le harnais à un cheval», tandis que le verbe anglais «to harness», qui a la même signification, possède aussi celle de «maîtriser une force et la faire servir à un usage particulier». L'expression (harnacher) un cours d'eau est un calque de l'anglais. Il faut dire AMÉNAGER. » (Dagenais, semaine du 26 septembre 1965)

\subsection{Les arguments employés pour critiquer un emploi}

En dernier lieu, nous avons analysé les arguments employés pour justifier les prises de position normatives des chroniqueurs. Les arguments qu'ils mettent de l'avant pour condamner un anglicisme peuvent être de diverses natures, comme le liste le tableau 2. Or la plupart des arguments ne sont employés qu'en de rares occasions dans notre corpus.

Tableau 2. Arguments employés pour condamner un anglicisme ${ }^{16}$

\begin{tabular}{|c|c|c|c|c|c|c|}
\hline & Lusignan & Fréchette & De Chantal & Dagenais & Roux & Bertrand \\
\hline Origine anglaise & 23 & 10 & 20 & 29 & 38 & 52 \\
\hline Sens français & 4 & 4 & 1 & 10 & 1 & 10 \\
\hline Dictionnaire & 0 & 0 & 3 & 0 & 6 & 5 \\
\hline Usage en France & 0 & 0 & 0 & 0 & 3 & 4 \\
\hline Logique de la langue & 0 & 0 & 0 & 3 & 0 & 1 \\
\hline Étymologie & 0 & 0 & 0 & 2 & 0 & 2 \\
\hline Anglicisme inutile & 0 & 0 & 0 & 0 & 2 & 1 \\
\hline Sens anglais & 0 & 0 & 0 & 1 & 0 & 1 \\
\hline
\end{tabular}

Dans l'ensemble, les chroniqueurs expliquent rarement leur démarche pour condamner un anglicisme:

16. Les chiffres du tableau désignent le nombre d'emplois pour lesquels un argument est employé. 
(8) «Défiez-vous de cet anglicisme : «Sous ces circonstances (under those circumstances)»; dites et écrivez : «dans ces circonstances». On peut reprocher cet anglicisme à la plupart de nos hommes politiques, ministres et députés. » (Lusignan, 6 mai 1884)

On observe ici que le chroniqueur n'explique pas pourquoi il juge qu'il faut éviter cet emploi ni la façon dont il est arrivé à cette conclusion ; il ne fait que donner l'expression française par laquelle il faut le remplacer. L'origine anglaise semble être l'unique raison pour laquelle Lusignan condamne l'emploi de "sous ces circonstances». En fait, l'origine anglaise d'un anglicisme est très souvent brandie comme argument pour condamner un usage, dans notre corpus comme dans d'autres: "Les chroniqueurs s'appuient parfois sur les origines d'un fait de langue pour le condamner (lorsqu'il s'agit d'un emprunt à l'anglais) ou pour le justifier lorsqu'il s'agit d'un héritage de France (v. Remysen 2009b) » (Remysen, 2011 : 57). Dans notre corpus, nous avons identifié huit arguments différents employés pour critiquer un anglicisme. Or pour tous les anglicismes qui composent notre corpus, $73 \%$ des cas sont critiqués seulement en raison de leur origine anglaise, ce qui nous démontre la force de cet argument.

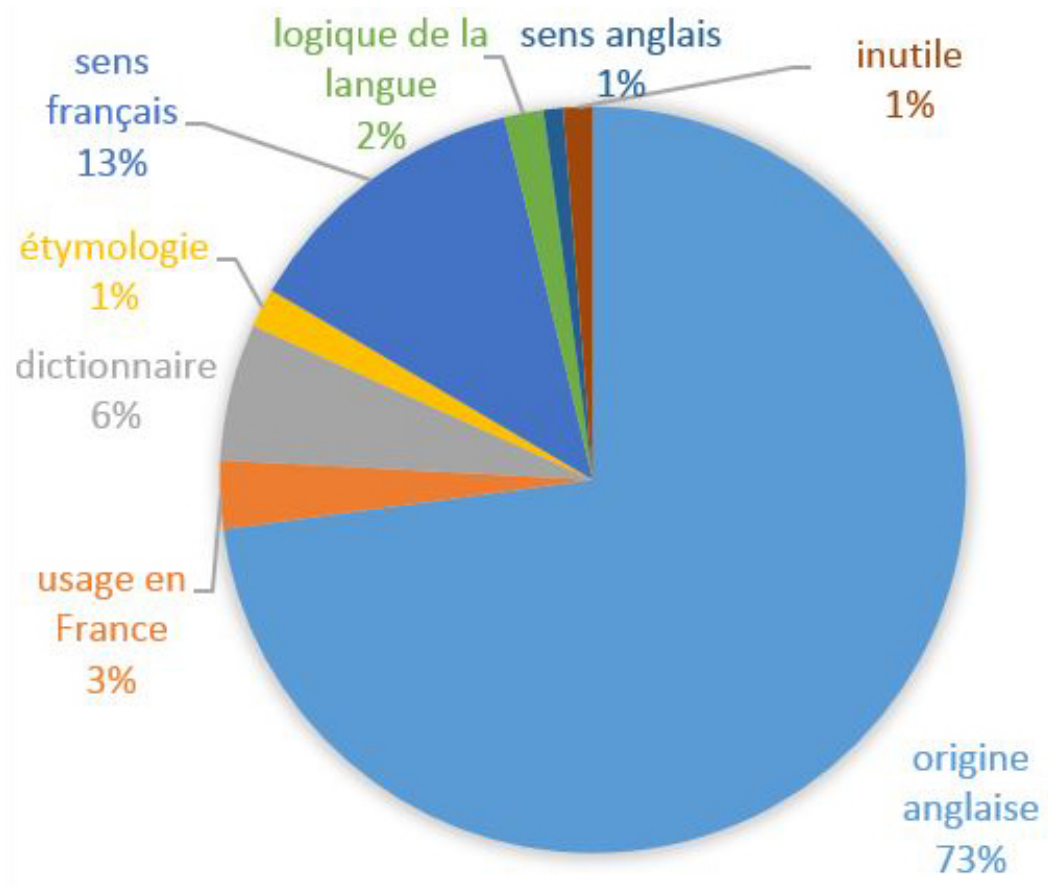

\section{Schéma 7. Pourcentage des arguments employés pour condamner un anglicisme}

Ainsi, près des trois quarts des anglicismes ne reçoivent aucune autre forme de procès que leur origine anglaise pour se faire condamner. L'extrait suivant d'une chronique de Bertrand illustre comment se présente l'argument de l'origine anglaise lorsqu'il est employé seul : 
(9) «C'est l'éléphant dans la pièce est un calque de l'anglais. Il n'existe pas d'expression imagée en français pour exprimer la réalité évoquée par cette locution. Au lieu de dire, par exemple, que la violence dans les foyers de personnes âgées, c'est l'éléphant dans la pièce, on dira que la violence dans les foyers de personnes âgées, c'est le problème dont on n'ose pas parler, que c'est le problème qu'on ne veut pas voir, que c'est le problème qu'on fait semblant d'ignorer ou que c'est l'évidence dont personne ne parle. » (Bertrand, semaine du 15 janv. 2018)

Bertrand présente l'expression calquée de l'anglais, précise qu'elle ne possède aucun équivalent imagé en français, puis propose une série d'expressions pour la remplacer. L'origine anglaise de l'expression est donc l'unique facteur menant à la condamnation de cet usage. C'est également le cas dans l'extrait suivant d'une chronique de Louis Fréchette:

(10) « II ne faut blâmer personne de parler l'anglais ; il faut bien se faire comprendre de ceux qui n'ont pas eu l'avantage ou le courage d'apprendre les deux langues. Ce qu'il faut blâmer, ce sont ceux qui se servent de l'anglais pour corrompre et défigurer notre langue, à nous. Ce sont eux, par exemple, qui vous diront, et qui écriront même, qu’un cheval a été « injurié », parce que les Anglais disent «injured» (blessé). » (Louis Fréchette, 28 octobre 1899)

Le mot injurier a un autre sens en français, soit le sens de insulter. II s'agit donc d'un anglicisme sémantique, et si Fréchette l'avait évoqué, nous aurions ajouté pour cet emploi commenté l'argument « sens français ». Or l'auteur ne fait mention que de l'origine anglaise de l'emploi, sans évoquer d'autre argument.

Le sens français d'un mot est justement le $2^{\mathrm{e}}$ argument mis de l'avant pour condamner un anglicisme dans notre corpus, loin derrière l'argument de l'origine anglaise. Cet argument s'applique aux emprunts de sens, pour lesquels l'emploi influencé par l'anglais ne respecterait pas le sens déjà existant en français du mot ou de l'expression en question :

(11) « «Apologie» signifie défense, justification, mais jamais excuse. On ne doit donc pas dire: «Je vous fais apologie», «ll me doit des apologies». En anglais, apology s'emploie dans le sens d'excuse ; le mot excuse est son seul équivalent en français. Que l’on se garde donc de cet anglicisme malheureusement trop répandu. » (Lusignan, 26 mai 1884)

On constate que les chroniqueurs ont peu recours au dictionnaire ou à d'autres sources de référence, comme l'OQLF, en tant qu'argument pour appuyer leurs condamnations - quoiqu'un chroniqueur pourrait consulter des dictionnaires sans le mentionner dans sa chronique. Dans notre échantillon, De Chantal, Roux et Bertrand invoquent quelques fois cet argument:

(12) « Le Nouveau Petit Robert mentionne qu'au Québec on emploie centre d'achats pour traduire shopping center. L'OLF recommande plutôt centre commercial. Ce dernier emploi paraît d'autant plus justifié que l'expression centre d'achats désigne déjà en français un «bureau central responsable des achats». » (Roux, 9 octobre 1997) 
Ici, Roux fait référence au dictionnaire le Nouveau Petit Robert ainsi qu'à la recommandation de l'OLF (Office de la langue française, ancien nom de l'Office québécois de la langue française). Il explique ensuite que l'expression centre d'achats désigne une autre réalité en français - il utilise donc également l'argument «sens français». Dans l'extrait suivant d'un billet de 1953, De Chantal énumère les ouvrages qu'il a consultés pour condamner « en autant que ». Il juge cette locution fautive, et émet l'hypothèse d'une influence de l'anglais sur la construction française :

(13) «Cette locution est fautive. Elle est sans doute née par contamination avec "en tant que"; de plus, l'influence de l'anglais « in as much as » n'est peut-être pas négligeable. On ne trouve "en autant que" dans aucun des bons dictionnaires ; Littré, Quillet, Larousse, Hatzfeld et Darmesteter, ou Bescherelle. » (De Chantal, 19 février 1953)

Dagenais et Bertrand font parfois appel à ce que nous avons appelé la logique de la langue française pour condamner un anglicisme :

(14) « Année de calendrier est un calque de l'anglais qui n'est pas conforme à la pensée française. » (Dagenais, semaine du 12 déc. 1965)

Les chroniqueurs évoquent occasionnellement l'étymologie du mot français comme argument pour condamner un emploi :

(15) «Arrivé en Angleterre, le melon y a gardé son nom français mais le p final est prononcé en anglais. Le mot ne remontant pas plus loin que 1791, c'est un anglicisme que l'on commet au Canada quand on y dit (can-ta-lou-pe) au lieu de can-ta-lou. » (Dagenais, semaine du 7 nov. 1965)

Ainsi, pour Dagenais, comme cantaloup est d'étymologie bien française, il devrait être prononcé sans le [p] final, prononciation anglaise. Un autre argument parfois évoqué pour critiquer un anglicisme est son « inutilité », c'est-à-dire le fait que l'emploi ne contribue pas à enrichir la langue française puisqu'il existe déjà un équivalent français :

(16) « L’anglicisme fact checking est inutile puisque le terme recommandé en français est la traduction littérale du terme anglais, en l'occurrence, vérification des faits. » (Bertrand, semaine du 30 oct. 2017)

Enfin, le sens anglais d'un emploi sert parfois d'argument aux chroniqueurs pour le condamner. L'emploi du mot ou de la tournure anglaise en français ne respecterait pas le sens dans la langue d'origine : 
(17) «En anglais to punch out, c'est pointer en quittant l'usine ou le bureau. Ce verbe n'a rien à voir avec le fait de se soustraire à un engagement. Dans le jargon des pilotes d'avions à réaction, to punch out exprime le fait d'appuyer sur le bouton du siège éjectable. Ici, comme on parlait du droit d'une mère porteuse à revenir sur sa décision, on aurait pu dire : la mère porteuse peut changer d'idée (se désister, se retirer du projet, mettre fin à l'entente) n'importe quand. » (Bertrand, semaine du 12 mars 2018)

Toutefois, ces trois derniers arguments ne sont que rarement utilisés et toujours en accompagnement de l'argument de l'origine anglaise.

\subsection{Arguments justifiant l'emploi d'un anglicisme}

Nous avons également examiné les arguments mis de l'avant lorsqu'un anglicisme était accepté par un chroniqueur. Cependant, en raison de l'approche normative prédominante dans notre corpus, ils sont trop peu nombreux dans notre corpus pour obtenir des résultats représentatifs ni pour les catégoriser. Mentionnons seulement que la plupart des quelques anglicismes acceptés dans notre corpus le sont sans qu'on ne donne de raison, comme c'est le cas avec magasin à rayons qui fait l'objet d'un commentaire chez Bertrand:

(18) « Magasin à rayons est le calque de l'anglais department store, mais ce terme est considéré comme un régionalisme acceptable. » (Bertrand, semaine du 27 nov. 2017).

Roux a une courte avance pour l'acceptation des anglicismes, et il présente parfois des motifs pour appuyer son jugement normatif. Dans la chronique qu'il consacre à attrition, mot « apparu en 1972 en français, vraisemblablement sous l'influence de son homonyme anglais » (Roux, 30 mars 1997), il fait état de sa recherche dans des ouvrages de référence et explique que cet anglicisme ne possède pas d'équivalent français :

(19) «Attrition: On le trouve dans le Petit Robert ainsi que dans le Multidictionnaire. Mais curieusement, il a disparu de la dernière édition de ces deux dictionnaires. Bien que son emploi soit contesté par certains auteurs, on voit mal par quoi on pourrait le remplacer, sinon par une périphrase. » (Roux, 30 mars 1997)

\subsection{Portrait des deux chroniqueurs contemporains}

Rappelons que notre corpus comprend deux chroniqueurs contemporains, soit Paul Roux et Guy Bertrand. Notons d'abord un point en commun entre les deux chroniques : ce sont celles qui commentent le plus d'anglicismes parmi les six analysées dans notre étude. Plus de la moitié des emplois commentés par ces deux chroniqueurs sont des anglicismes, soit 52\% pour Roux et 56 \% pour Bertrand. 
Roux est le chroniqueur qui accepte le plus grand nombre d'anglicismes dans notre corpus ${ }^{17}$ et qui en condamne le moins; de même, Roux est le chroniqueur le plus nuancé de notre corpus, même s'il se situe tout de même dans la tendance générale à commenter et critiquer les anglicismes. Un cinquième $(21 \%)$ des anglicismes qu'il critique reçoit un verdict nuancé. Par ailleurs, même s'il utilise parfois l'argument « origine anglaise » seul pour condamner un usage, il est celui qui rend le plus compte de ses recherches dans les dictionnaires; il compare avec l'usage en France et lorsqu'il condamne, il ne le fait pas toujours de manière catégorique. L'extrait suivant fait intervenir plusieurs de ces éléments :

(20) «Ce calque de proactive est à la mode tant dans le vocabulaire de la psychologie que dans celui de la gestion, où on lui donne le sens de « qui prend les devants », « qui agit sur des faits à venir ». Proactif n’a pas encore reçu l'aval des dictionnaires usuels, sans doute parce qu'il est peu utile. » (Roux, $1^{\text {er }}$ mai 1999)

Pour sa part, Guy Bertrand est le chroniqueur du corpus qui commente le plus grand nombre d'anglicismes, 56 sur 100 emplois commentés, et il est aussi celui qui en condamne le plus, soit 52 des 56 anglicismes en question, ce qui représente $93 \%$. Il est aussi le chroniqueur le plus catégorique au moment de condamner un anglicisme : sa condamnation est catégorique pour 51 anglicismes critiqués sur 52, ce qui représente $98 \%$ des cas, et l'origine anglaise est le seul argument qu'il met de l'avant pour appuyer une condamnation dans 58 \% des cas, c'est-à-dire pour 30 anglicismes, comme l'illustrent les exemples suivants:

(21) «Bien qu'il soit courant dans le jargon des musiciens de chez nous, le mot band doit être considéré comme un anglicisme à éviter. » (Bertrand, semaine du 4 déc. 2017)

(22) «En anglais, wannabe est un terme familier correspondant à la contraction de want to be qui signifie qui désire être. En anglais, on utilise wannabe en parlant d'une personne qui souhaite accéder à un poste ou à un statut quelconque sans nécessairement avoir tout ce qu'il faut pour y parvenir. Ici, il aurait suffi de dire : elle rêve de devenir comédienne, elle aspire à devenir comédienne ou elle voudrait devenir comédienne. » (Bertrand, semaine du 4 déc. 2017)

Dans ce dernier extrait, Bertrand condamne wannabe en raison de son origine anglaise, même s'il ne lui reconnaît pas d'équivalent français, et même s'il est surtout courant dans la langue orale ou familière plutôt que dans un registre standard - ce qui est également le cas de band, dans l'extrait 21. Ceci nous amène à évoquer une tendance chez Bertrand à ne pas tenir compte des registres lorsqu'il propose des équivalents français à un anglicisme. Il suggère, par exemple, de remplacer l'expression familière ou populaire «ne pas se prendre pour un 7 up flat » par «Elle se croit sortie de la cuisse de Jupiter ». Cet aspect pourrait faire l'objet d'une étude plus approfondie.

17. Après De Chantal, dont l'écart créé par un billet spécifique a été expliqué dans la section 3.2. 


\section{Quelques conclusions}

Le schéma suivant représente la proportion d'anglicismes commentés par chacun des chroniqueurs de notre corpus:

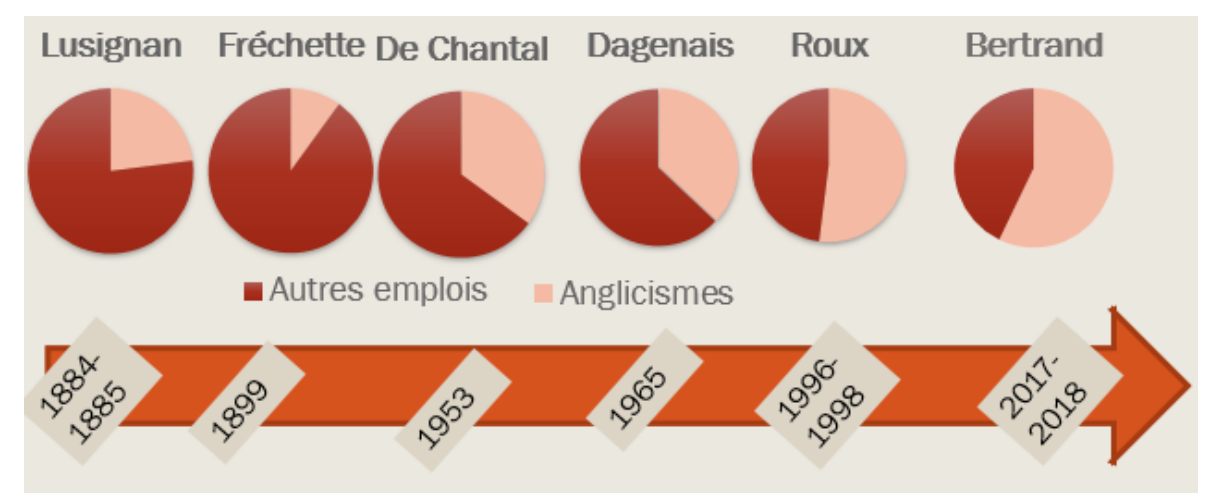

Schéma 8. Proportion d'anglicismes commentés par chroniqueur

On constate que l'anglicisme demeure un sujet de préoccupation normative important chez les chroniqueurs de langue contemporains, qui y consacrent plus de place que certains de leurs prédécesseurs. À la lumière de nos analyses, les chroniqueurs contemporains ne semblent se démarquer par aucun autre critère. Les anglicismes formels sont encore le type d'anglicismes les plus critiqués. L'origine anglaise d'un emploi demeure un critère suffisant pour condamner un anglicisme dans la plupart des cas, et on ne rend pas souvent compte des autres arguments qui auraient éventuellement permis au chroniqueur de prendre une décision normative par rapport à un anglicisme.

Les chroniqueurs contemporains sont-ils décalés par rapport aux préoccupations des locuteurs ordinaires $^{18}$ ? Font-ils montre d'un plus grand purisme ? Leurs chroniques ne font-elles que refléter les préoccupations des locuteurs qui les consultent, qui ont au départ une préoccupation normative ? Le rôle de gardiens de la norme des chroniqueurs de langue les amène peut-être à conserver une plus grande prudence envers les anglicismes, les positionnant en porte-à-faux par rapport aux locuteurs ordinaires.

On peut également se demander pourquoi les autres éléments sur lesquels les chroniques se penchaient auparavant, tels les archaïsmes, les barbarismes ou les éléments syntaxiques, sont moins présents dans les chroniques d'aujourd'hui. Ces éléments considérés comme fautifs sont-ils moins nombreux aujourd'hui, éventuellement en raison du plus haut taux de scolarisation comparativement à la mi-XIXe siècle et au début du XXe siècle, alors que les campagnes de rectification langagière sont à leur plus fort? D’autres études permettraient de dresser un portrait plus complet de la situa-

18. Rheault (2010) a étudié la perception des locuteurs n'évoluant pas dans un domaine lié à la langue envers la langue en général et les anglicismes en particulier. Elle arrive à la conclusion que ces locuteurs ont une attitude plutôt neutre envers les anglicismes. 
tion. Il n'en reste pas moins que les chroniqueurs de langue, pour leur part, semblent avoir toujours «peur» des anglicismes. 


\section{Références}

\section{Corpus}

Bertrand, Guy (2017-2018), Le français au micro, disponible sur http://linguistique.radio-canada.ca/ category/le-francais-au-micro. [Page consultée entre octobre 2017 et février 2018.]

De Chantal, René (1953-1963), « Défense et illustration de la langue française », Le Droit, Gatineau, Ottawa.

Dagenais, Gérard (1965-1966), «Comment dites-vous ? », La Patrie, Montréal.

Fréchette, Louis (1893-1903), «À travers le dictionnaire et la grammaire, corrigeons-nous! », La Presse, Montréal.

Lusignan, Alphonse (1884-1885), « Fautes à corriger : une chaque jour », La Patrie, Montréal.

Roux, Paul (1996-1999), «Le mot du jour », La Presse, Montréal.

\section{Sources citées}

Boisvert, Lionel (1987), «L'anglicisme, voilà l’ennemi ? », Québec français, n 65, p. 24-26.

Bouchard, Chantal (1989), «Une obsession nationale : l'anglicisme », Recherches sociographiques, vol. 30, n²1, Québec, Département de sociologie, Université Laval, p. 67-90.

Bouchard, Chantal (1999), On n'emprunte qu'aux riches: la valeur sociolinguistique et symbolique des emprunts, Montréal, Fides.

Dagenais, Gérard (1959), «Réflexions sur nos façons d’écrire et de parler », Le Devoir, Montréal, lundi 20 avril 1959, p. 14 et 10.

Gagné, Frédérick, Claude Verreault et Louis Mercier (2004), «La base de données textuelles ChroQué : un nouvel outil pour élargir la description du français en usage au Québec », dans Louis Mercier (dir.) avec la collaboration d'Hélène Cajolet-Laganière, Français du Canada-français de France. Actes du sixième Colloque international d'Orford (Québec), Tübingen, Max Niemeyer Verlag («Canadiana Romanica »), p. 247-262.

Gagné, Frédérick et Claude Verreault (2011), «Louis Fréchette », dans Claude Verreault, Louis Mercier et Wim Remysen (dir.), Chroqué : base de données textuelles de chroniques québécoises de langage, disponible sur http://catfran.flsh.usherbrooke.ca/chroque. [Page consultée le 15 janvier 2019.]

Loubier, Christiane (2011), De l'usage de l'emprunt linguistique, Québec, Office québécois de la langue française. 
Manseau, Joseph Amable (1881), Dictionnaire des locutions vicieuses du Canada avec leur correction: suivi d'un dictionnaire canadien, Québec, J. A. Langlais.

Mercier, Louis (2008), «À la découverte des particularismes canadiens et de leur origine : la lexicographie québécoise à l'époque des glossaires (1880-1930) », dans Monique C. Cormier et JeanClaude Boulanger (dir.), Les dictionnaires de la langue française au Québec: de la Nouvelle-France à aujourd'hui, Montréal, Les Presses de l'Université de Montréal, p. 61-98.

Mercier, Louis, Wim Remysen et Hélène Cajolet-Laganière (2017), "Québec », dans Ursula Reutner (dir.), Manuel des francophonies, Berlin, De Gruyter, p. 277-310.

Pellerin, Josiane (2007), Louis-Philippe Geoffrion, chroniqueur de langage : le thème de l'anglicisme dans les «Zigzags autour de nos parlers» (1923-1927), mémoire de maîtrise, Sherbrooke, Université de Sherbrooke.

Prévost, Geneviève (1995), "Les chroniques de langage d’Étienne Blanchard (1883-1952) », dans Julie Audette, Marc-André Bélanger, André Bourcier, Isabelle Dion, Pierre Larrivée, Julie Nicole, François Pichette et Emily Rosales (dir.), Actes des ge Journées de linguistique, Québec, Centre international de recherche en aménagement linguistique (« B-201»), p. 117-121.

Remysen, Wim (2009), Description et évaluation de l'usage canadien dans les chroniques de langage: contribution à l'étude de l'imaginaire linguistique des chroniqueurs canadiens-français, thèse de doctorat, Québec, Université Laval.

Remysen, Wim (2011), «L'application du modèle de l'imaginaire linguistique à des corpus écrits : le cas des chroniques de langage dans la presse québécoise », Langage et société, n 136, p. 47-65.

Remysen, Wim et Bianca De la Fontaine (2013), "L'héritage du Glossaire du parler français au Canada (1930) dans les chroniques de langage parues dans la presse canadienne-française : un aperçu », dans Anne-Marguerite Fryba, Roberto Antonelli et Bernard Colombat (dir.), Actes du XXVII Congrès international de linguistique et de philologie romanes (Nancy, 15-20 juillet 2013). Section 15 : Histoire de la linguistique et de la philologie, Nancy, Laboratoire ATILF, p. 157-170.

Remysen, Wim et Alexandra Tremblay-Desrochers (2011), « Gérard Dagenais », dans Claude Verreault, Louis Mercier et Wim Remysen (dir.), Chroqué : base de données textuelles de chroniques québécoises de langage, disponible sur http://catfran.flsh.usherbrooke.ca/chroque. [Page consultée le 15 janvier 2019.]

Rheault, Amélie-Hélène (2010), Analyse argumentative du discours épilinguistique au Québec : les lieux communs comme indicateurs de normes, thèse de doctorat, Sherbrooke/Louvain, Université de Sherbrooke/Université Catholique de Louvain.

Steuckardt, Agnès (2008), «L'anglicisme politique dans la seconde moitié du $18^{e}$ siècle. De la glose d'accueil à l'occultation », Mots. Les langages du politique, n 82, p. 9-22.

Verreault, Claude (2011), «Alphonse Lusignan», dans Claude Verreault, Louis Mercier et Wim Remysen (dir.), Chroqué: base de données textuelles de chroniques québécoises de langage, disponible sur http://catfran.flsh.usherbrooke.ca/chroque. [Page consultée le 15 janvier 2019.] 
Verreault, Claude, Louis Mercier et Wim Remysen (dir.) (2011), Chroqué: base de données textuelles de chroniques québécoises de langage, disponible sur http://catfran.flsh.usherbrooke.ca/chroque. 